\title{
Hepatic manifestations during amoebic dysentery
}

\author{
S. RAMACHANDRAN \\ M.D., M.R.C.P., M.R.C.P.E. \\ R. DE SARAM \\ M.B., B.S. \\ C. N. A. Rajapakse \\ S. Sivalingam \\ M.B., B.S. \\ M.B., B.S., Ph.D., F.R.C.Path. \\ Colombo North General Hospital, Ragama, and the Medical Research Institute, \\ Colombo, Sri Lanka
}

\begin{abstract}
Summary
A variety of hepatic manifestations may occur during attacks of acute amoebic dysentery, conforming to clearly defined clinical groups.

The incidence of definite hepatic involvement presenting as cases of hepatic amoebiasis with or without demonstrable pus or with tender hepatomegaly was $57.5 \%$.

While it is possible that the cases presenting with hepatic manifestations without demonstrable pus are due to small deep-seated abscesses, the non-specific nature of the histological lesions observed in the study and the inability to demonstrate the presence of amoebae in the lesions support the contention that these hepatic manifestations may represent a nonspecific reaction in the liver to amoebic ulceration of the colon.

Chest radiology may be helpful in confirming the clinical evidence of hepatic involvement but the leucocyte count, ESR, and the serum enzymes are of limited value in indicating both its presence and type.

Patients manifesting hepatic involvement during attacks of amoebic dysentery should be adequately treated with tissue amoebicidals. This therapeutic approach would be useful in reducing the incidence of hepatic amoebiasis.
\end{abstract}

\section{Introduction}

In order to study the relationship between intestinal and hepatic amoebiasis it would seem necessary to observe whether hepatic involvement occurs during attacks of acute amoebic dysentery. This paper reports the incidence and the different types of hepatic manifestations occurring during acute and subacute amoebic dysentery.

\section{Patients, materials and methods}

Observations were based on a study of twentyeight consecutive cases of acute and subacute amoebic dysentery where active motile amoebae contain- ing ingested red cells were seen during microscopic examination of stools in every case. The age of the patients ranged from 12 to 85 years with a mean of 39 years, and twenty-seven of the cases were male subjects. All the patients were examined clinically and this was followed by haematological, biochemical and radiological studies. Percutaneous liver biopsy was performed in twenty-two of the cases. The necropsy appearances in the only case with a hepatic abscess will be described.

The duration of the diarrhoea ranged from 2 to 60 days with a mean of 18 days. A previous history of dysentery was obtained in twelve cases $(43 \%)$; these attacks occurred from 1 month to 5 years prior to the present episode.

\section{Results}

The relevant clinical features in these cases are given in Table 1 . In respect to evidence of hepatic involvement it appeared that the patients fell into one of five clearly defined clinical groups.

TABLE 1. Clinical features in patients with acute and subacute dysentery

\begin{tabular}{lc}
\hline Feature & No. of cases \\
\hline $\begin{array}{l}\text { Fever } \\
\text { Right hypochondrial pain }\end{array}$ & $11(39 \%)$ \\
Downward hepatomegaly & $21(75 \%)$ \\
$1 \cdot 5 \mathrm{~cm}$ below costal margin & $9(32 \%)$ \\
$3 \mathrm{~cm}$ below costal margin & $4(14 \%)$ \\
$4 \cdot 5 \mathrm{~cm}$ below costal margin & $2(7 \%)$ \\
$\quad$ Total & $15(53 \%)$ \\
Intercostal and subcostal tenderness & $18(64 \%)$ \\
(mild to moderate) & $1(3.5 \%)$ \\
Clinical groups & $7(25 \%)$ \\
(1) Hepatic amoebiasis with pus (abscess) & $8(29 \%)$ \\
(2) Hepatic amoebiasis without pus & ('amoebic hepatitis') \\
(3) Tender hepatomegaly & $3(10 \cdot 5 \%)$ \\
(4) Hepatic tenderness without hepatic & $9(32.5 \%)$ \\
(5) No clinical hepatic involvement &
\end{tabular}


Group 1. Hepatic amoebiasis with demonstrable pus (hepatic abscess)

One case $(3.5 \%)$. This patient, a male, 38 years of age, presented with acute amoebic dysentery and severe diffuse abdominal pain. There was no downward hepatic enlargement or hepatic tenderness. He died of severe toxaemia and at necropsy there was a large, thick-walled chronic abscess 5 in. in diameter in the left lobe of the liver which had ruptured, causing a diffuse peritonitis. The colon showed extensive amoebic ulceration. Amoebae were seen both in the colonic mucosa and in the wall of the hepatic abscess during microscopic examination (Fig. 1).

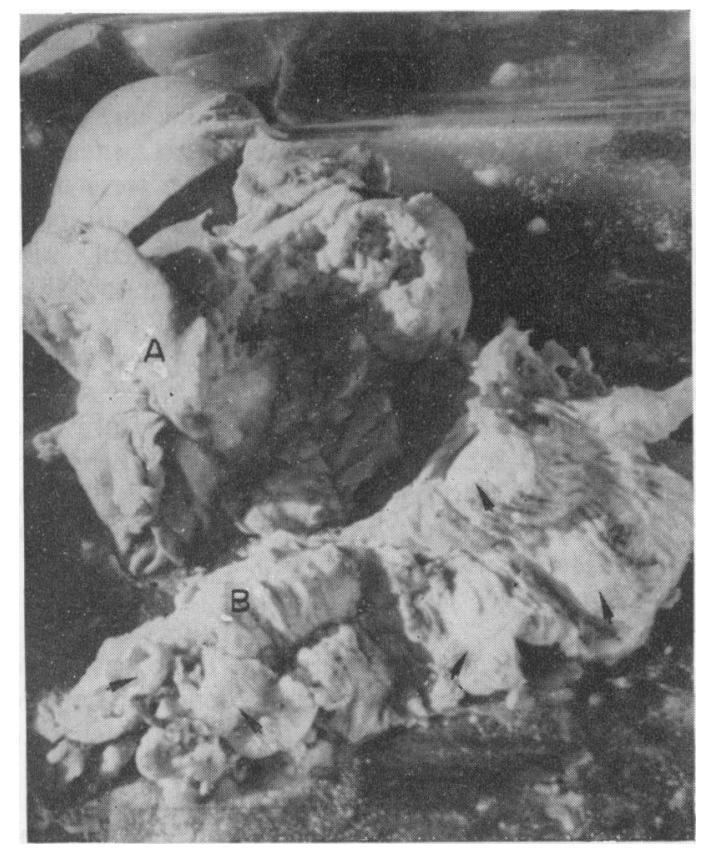

FIG. 1. Shows the necropsy appearances in the case with a liver abscess, (A) the liver with a large abscess and: (B) the colon with extensive amoebic ulceration (arrowed).

\section{Group 2. Hepatic amoebiasis without demonstrable pus (amoebic hepatitis)}

Seven cases $(25 \%)$. These cases were found to satisfy more than three of the criteria namely, an enlarged tender liver, previous history of dysentery, leucocytosis, radiological changes and a good response to therapy (Ramachandran, Sivalingam \& Perumal, 1972).

\section{Group 3. Hepatic enlargement and tenderness}

Eight cases $(29 \%)$. These eight cases had mild hepatic enlargement with tenderness but did not satisfy more than three of the above mentioned criteria.

\section{Group 4. Hepatic tenderness without hepatomegaly}

Three cases $(10.5 \%)$. There was mild to moderate hepatic tenderness without clinical or radiological evidence of hepatic enlargement.

\section{Group 5. No evidence of hepatic involvement}

Nine cases $(32 \%)$.

Thus, sixteen patients $(57.5 \%)$ by belonging to the Groups 1-3 had definite clinical evidence of hepatic involvement.

\section{Haematological and biochemical changes}

A leucocytosis occurred in $54 \%$ of the cases. While it was present in all the cases in Groups 1 and 2 it was also inconstantly observed in patients of the remaining groups. An elevation in the ESR of over $20 \mathrm{~mm}$ in the first hour was observed in $77 \%$ of the cases. Once again it was a constant feature in Groups 1 and 2 but some of the highest values were seen in patients of the remaining groups. Elevation in the serum bilirubin and the serum enzymes were minimal and when present they were noted in patients belonging to all the different groups (Fig. 2).

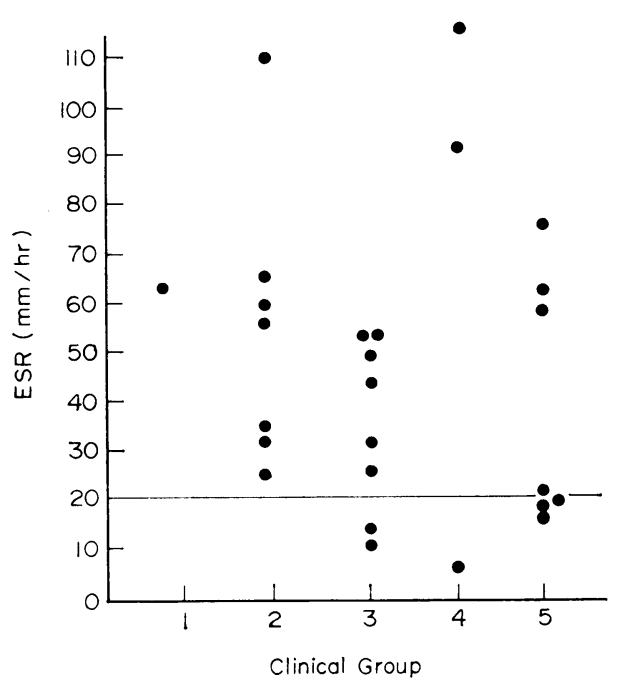

Fig. 2. Values for the ESR (twenty-six cases) in the different groups. While the cases in the Groups 1 and 2 had values of over $20 \mathrm{~mm}$ in the first hour some of the highest values were noted in the remaining groups.

\section{Radiological changes}

In the only patient with an hepatic abscess there was an elevation of the left hemidiaphragm while two of the cases in Group 2 had a significant elevation of the right dome of the diaphragm. Mild 
pleuro-pulmonary reactions inconstantly seen in the different groups was noted in nine patients $(32 \%)$.

\section{Hepatic histology}

Liver biopsy studies revealed a variety of nonspecific lesions. There was lymphocytic infiltration $(41 \%)$, portal tract infiltration $(62 \%)$, focal necrosis $(14 \%)$, granulomatous lesions $(18 \%)$ and fatty change $(50 \%)$. These changes were inconstantly seen among patients belonging to the different clinical groups. Striking was the observation that, apart from the only patient with an hepatic abscess, amoebae were not demonstrated during histological examination of the liver biopsies (Figs. 3 and 4).

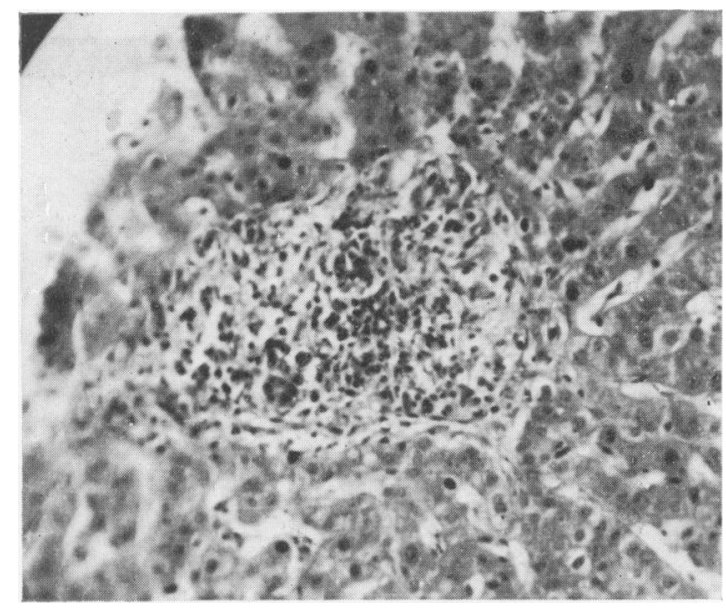

Fig. 3. Portal tract showing a granulomatous change. No amoebae were demonstrable. $(\times 85)$

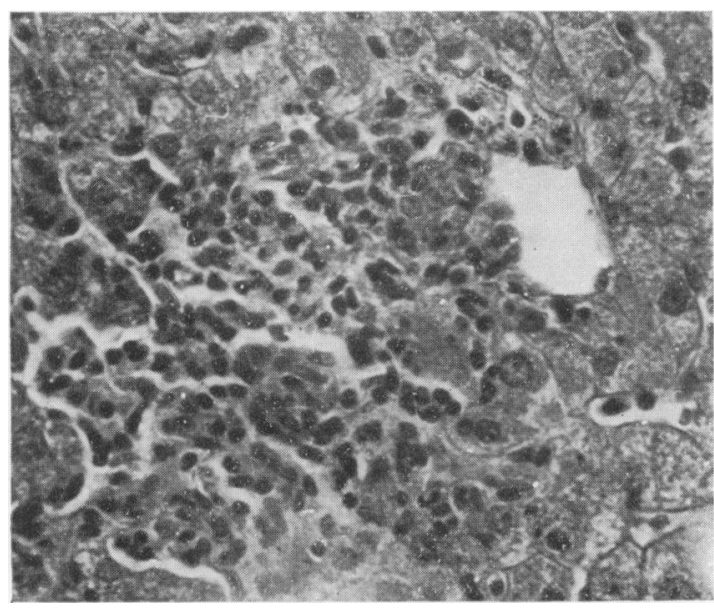

FIG. 4. An area of liver cell necrosis (perivenous) with mononuclear cell infiltration and early microabscess formation. No amoebae were demonstrable. $(\times 170)$

\section{Discussion}

It is clearly evident that a variety of hepatic manifestations could occur during attacks of acute amoebic dysentery. Definite clinical evidence of hepatic involvement was present in $57.5 \%$ of the cases. Though amoebic abscesses of the liver usually become manifest after a variable period following intestinal infections with Entamoeba histolytica (Craig, 1944), there is no doubt that a hepatic abscess may be present even during a episode of acute or subacute amoebic dysentery (Futcher, 1903; Banker, 1947). Hence, it would become necessary to exclude the presence of an hepatic abscess during the care of patients with amoebic dysentery, where the bowel symptoms may dominate the clinical picture. Hepatic involvement without demonstrable pus was observed in a proportion of the patients in this study. It is not often possible to demarcate by clear-cut signs and symptoms cases where pus may be demonstrated by therapeutic aspiration (proven hepatic abscesses) from the group of cases where pus may not be demonstrated (Ramachandran, Sivalingam \& Perumal, 1973). This is especially so in the case of small hepatic abscesses. Thus while it is possible that a proportion of the cases of hepatic involvement without demonstrable pus are in fact cases with small deep-seated abscesses (Lamont \& Pooler, 1958; Ramachandran et al., 1973), the non-specific nature of the histological lesions observed and the absence of amoebae in the lesions support the contention that the hepatic manifestations may only be a nonspecific reaction occurring as a consequence of amoebic ulceration of the colon.

Radiology of the chest is useful in confirming the clinical evidence of hepatic involvement during attacks of amoebic dysentery and for this purpose both posterio-anterior and right lateral views of the chest should be taken (Ramachandran, Jayawardena \& Perumal, 1971). In contrast the leucocyte count, ESR and the serum enzymes appear to be of limited value in both indicating the presence of hepatic involvement and in differentiating the type of hepatic involvement.

From the clinical standpoint it would appear that in patients presenting with amoebic dysentery, evidence of hepatic involvement should be specifically noted and when present such cases should be adequately treated with tissue amoebicidals. Such a therapeutic approach no doubt would be useful in reducing the incidence of hepatic amoebiasis.

\section{References}

Banker, D.D. (1947) Amoebic abscess of the liver. Tropical Diseases Bulletin, 45, 338.

Craig, C.F. (1944) The Etiology and Diagnosis and Treatment of Amoebiasis. Williams and Wilkins Co., Baltimore.

Futcher, T.B. (1903) A study of the cases of amoebic dysentery occurring at the Johns Hopkins Hospital. Journal of the American Medical Association, 42, 480. 
Lamont, N.McE. \& Pooler, N.R. (1958) Hepatic amoebiasis. Quarterly Journal of Medicine, 27, 390.

Ramachandran, S., Jayawardena, D.L.N. \& Perumal, J.R.A. (1971) Radiological changes in hepatic amoebiasis. Postgraduate Medical Journal, 47, 615.
Ramachandran, S., Sivalingam, S. \& Perumal, J.R.A. (1972a) Hepatic amoebiasis in Ceylon. Journal of Tropical Medicine and Hygiene, 75, 34.

Ramachandran, S., Sivalingam, S. \& Perumal, J.R.A. (1973) Concepts in hepatic amoebiasis. Journal of Tropical Medicine and Hygiene, February. 